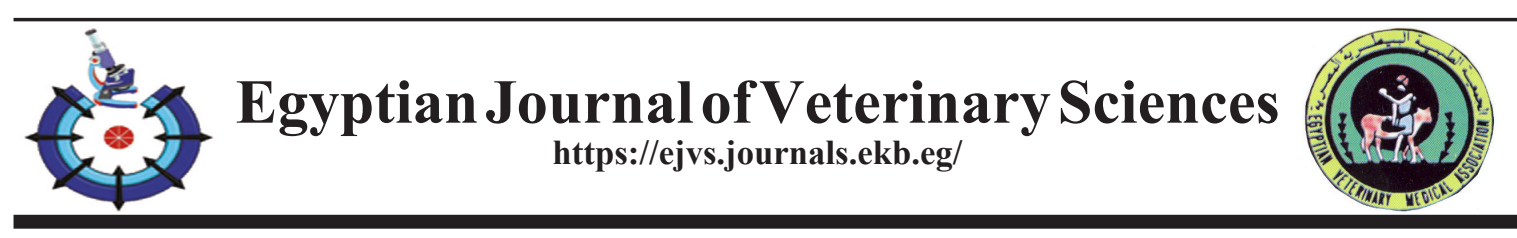

\title{
Epidemiological aspects of poisoning Infectious-toxic bacteria of botulism on food poisoning in Iran; A review study
}

Halleh Mirbehresi ${ }^{1}$, Mohadeseh Pirhadi ${ }^{2}$,Samira Shokri ${ }^{2}$ and Alireza Esmaeili ${ }^{{ }^{*}}$ ${ }^{1}$ Assistant Specialist in Internal Neurology and Student Research Committee, Ahvaz Jundishapur University of Medical Sciences, Ahvaz, Iran.

${ }^{2}$ Department of Environmental Health Engineering, Division of Food Safety \& Hygiene, School of Public Health, Tehran University of Medical Sciences, Tehran, Iran. ${ }^{3}$ Assistant Professor of Hematology and Oncology, School of Medicine, Ilam University of Medical Sciences, Ilam, Iran.

B OTULISM is a rare neuroparalytic syndrome, caused by a neurotoxin produced by bacteria of the genus Clostridium. Signs and symptoms of food botulism begin 12 to 36 hours after the toxin enters the bloodstream .Clostridium botulinum is one of the most common life-threatening agents worldwide, producing the botulinum neurotoxin (BoNT). Poisoning is caused by the consumption of a highly toxic exotoxin produced during the growth of microorganisms in food. The growth of Clostridium botulinum strains and their production of toxins in vacuum processed foods have received special attention. Symptoms of botulism may appear 12 to 72 hours after eating foods that contain toxins, such as canned tuna, local dairy products, and home-made or commercial foods. Symptoms of poisoning include nausea, vomiting, bruising, dizziness and headache, dry skin, sore throat, constipation, mild fever (or no fever), muscle paralysis, diplopia, and eventually respiratory problems and death. On the other hand, botulinum neurotoxin is used in medicine and psychiatry. BoNT is involved in the management and control of many diseases such as depression and Parkinson's, dermatitis such as psoriasis, as well as in the beauty and elimination of facial skin wrinkles.

Keywords: Clostridium botulinum, Botulism, Nervous system, Poison in the blood, Foods.

\section{Introduction}

Diverse types of marine foods have been consumed as healthy nutrients all over the world. Therefore regarding daily-increasing in aquatics, more attention has been also intended to their health [1]. Sake of having high-level calorie and protein as well as the presence of unsaturated fat in the form of omega 3 has been of particular significance in people's diet all over the world [2]. High ability-spoilage of fishes caused to quality-keeping of fresh-fish has been converted to one of the important problems from point of fish-industry and consumers. In this case canning, salting, and smoking of food substances as well as intending to self-life have been important [3]. Based on studies local food consumption that has been produced from smoked products and salted fishes in beach areas of Caspian sea because lack of attention to hygienic principles has been caused to food-born botulism poisoning and even death [4]. Some factors caused to increasing the contaminating-ability with clostridium bacteria and botulism toxin including of food-canning, un appropriate-keeping cans, unsuitable cooling, contamination sake of exudation and deterioration before producing-process, inappropriate cantransportation. Clostridium bacteria has been frequently found in the soil and water near beaches in most parts of the world, in addition to exudate eight classes of toxins. Regarding the exudated toxin-class, this bacteria has been included in 8 various types that have been called from A to $G$ [5].

*Corresponding author: Dr. Alireza Esmaeili, E-mail: esmaeili.alireza@yahoo.com

(Received 14/07/2021; accepted 22/08/2021)

DOI. 10.21608/ejvs.2021.83742.1246

C2022 National Information and Documentation Centre (NIDOC) 
Botulism is a kind of deadly paralytic disease that has been mainly caused by clostridium botulinum, meanwhile, this toxin has been known as the most powerful toxin so far [6]. Sake of the high death potential and the possibility of bioterrorism-risk via main distribution of this toxin in food-source has been reported as an emergency in general hygiene and world-security that each milligram of pure toxin could destroy half of 30 million of micepopulation $[7,8]$. This food poisoning in the form of the neuroparalytic syndrome has been often accompanied by side effects and disorders in the stomach and intestines [9]. Signs and symptoms of food botulism begin 12 to 36 hours after the toxin enters the bloodstream[9]. The main mechanism of botulism has been in the connection-point of the nerves to muscles in the end-membrane of the synaptic perineum. In addition to inhibition of Acetylcholine, neurotransmitter release is in the presynaptic nerve terminals that caused to decreasing in muscle- fiber activity, paralytic, as well as muscle-losing that has been basic application in botox makeup. After neurotoxin was connected to nerve cell receptors via endocrine cells has been located inside the membrane as well as decomposition of proteolytic proteins of synaptobrevin related to the terminal cell of the cholinergic nerve blocks the activity of extra chemical cell of Ach. The proteins are necessary to anchoring vesicles containing Ach in the presynaptic membrane. Without this connection, there is no possibility to release Ach in the synaptic cleft so the nervous structure would be paralyzed [10-12]. As well as it is accompanied by some symptoms such as diplopia, ptosis, dysphagia, oral dryness, extreme muscle weakness, and other nervous manifestation [13]. In addition to because E-type of clostridium botulinum among aquatics has been assigned the most part, as well as grows in the low temperature so has the growthability in the refrigerator-temperature. Caution to consuming fishes and other aquatics are more essential. In some countries such as Scandinavia, especially Denmark and Japan more than $60 \%$ of diseases resulting from fish-consuming have been dedicated to the salted and smoked kinds of E-type $[14,15]$. Center of disease control and prevention of USA has been claimed that annually almost 5.2 million diseases, 45826 cases of hospitalizations, 1458 cases of death have been caused via known pathogenic bacteria [16]. Studies have been revealed that the most food-contaminating agents with animal origin have been assigned to these bacteria respectively including fish-spawn
$30.9 \%$, salted fishes $23.4 \%$, home-made cheese $19.6 \%$, industrial can $7.5 \%$ that from point of the location-producing of foods $87.8 \%$ are homemade and $4.7 \%$ are industrial [17]. To considering different diseases in every country from point of distinguishing the contamination source what is extremely vital is the rate of disease outbreak in diverse areas of the country. The goal of this study also has been to consider the epidemiological aspects of food-borne poisoning caused by botulism and its effects on the nerve system in Iran.

\section{Method for review}

This study was conducted as a review article of articles published between 1980 and 2021. For this purpose, based on searches in Magiran, Google Scholar, SID, Scopus, PubMed, Science Direct, and ISI databases, articles were founded with the keywords including, Botulism, Clostridium botulinum, toxin consumed, marine foods.

\section{Results and Discussion}

Botulism has been known as one of the most dangerous and fatal poisonings. The main agents of this disease are neurotoxins of Clostridium botulinum [18]. The incidence of botulism may depend on macroeconomic indicators that affect people's eating habits. The incidence of botulism has grown during economic crises [19]. The distribution of different species of $\mathrm{C}$. botulinum has changed over the past 25 years (E.g., decrease in type B and increase in type E), which can be associated with an increase in canned fish products and a reduction in the use of canned meat [19]. Regarding the right process of canning and not consuming homemade cans, salted or smoked fishes are vital factors to preventing this poisoning. To considering the entrance-way of this toxin to food baskets human has a vital role from point of the high possibility death-event via its poisoning. Neurotoxins of clostridium botulinum especially A-type are the most toxic substances known in the world, as has been estimated one gram of pure A-type is enough to kill 100 million individuals [20]. A lot of reports from diverse parts of the world are based on separating clostridium botulinum or has been demonstrated food-born poisoning of botulism sake of consuming marine foods. researches have been conducted between 1920 and 2014 exposed that half of 197 outbreaks in the world were caused by A-type of clostridium toxins, meanwhile, the paralysis of the respiratory system has been relatively high [21]. Based on 
statistics about food outbreaks that have been reported in Iran, the reason for $2.8 \%$ of cases was botulism [22]. The results of the Centers for Disease Control and Prevention (CDC) have been reported 2037 suspected botulism caused by food in Iran between 2007 and 2017. Totally 252 cases $(12.3 \%)$ of botulism have been proved caused by food. The average annual incidence in every 100000 Iranian natives has been dedicated 7.1 cases to men and 3.3 cases to women. $19 \%$ of botulism cases were fatal [23]. 341 suspectedbotulism cases have been recorded in Iran between 2003 and 2007 that the most and the least cases have been respectively related to 2007 (28.86\%) and $2003(12.6 \%)$. Any cause of the disease has not been caused to death in 2006 and 2007. While 4 cases of death sake of food-borne poisoning of botulism and damaging to nerve cells had been recorded between 2003 and 2005. Among considered provinces, the most and the least poisoning cases have been respectively related to Gillan with 90 cases $(26.39 \%)$ and western Azerbaijan with 4 cases (1.17\%) [23]. Based on the Afshani report (2004) all suspected botulism cases in Iran between 1995 and 2003 were caused by food-consuming. Exported epidemiological data has been revealed that disease cases from point of geographical distribution have been respectively dedicated to Ghazvin (14\%), Golestan (5.2\%), Gillan (5.22\%), Zanjan (12\%) [17]. Spawn and salted fish with $31.08 \%$ cases have been known as the most frequent food-substance causing botulism. Reported cases of botulism sake of consuming salted spawn in Rasht in 2005 has been revealed that toxin-density of clostridium botulinum in the salted spawn of common crap fish is in the rate that has been able rapidly to cause to reveal symptoms of botulism. As well as salted spawn has been produced via traditional way has been contaminated by botulism, so the sake of muscle-paralysis of the respiratory system has occurred one case of death [4]. 116 cases of botulism poisoning have been recorded in Japan during recent 10 years that the most cases have been related to fermented products via traditional ways [24]. According to the study of Torense et al., the most important factor causing food-borne botulism poisoning in Argentina has been consuming homemade food-canned [25]. In European Union member states from 1992 to 2002, a total of 812 food-borne botulism poisoning cases have been recorded. Meanwhile, the incidence rate of this disease has been intended to decrease during these 10 years [25]. Un appropriate ways to food-consuming at home, un sufficient cooking, unsuitable saving, lack of adequate hygiene have been the most frequent agents causing botulism in home-made foods [26]. Considering one outbreak case of A-type botulism in one family member after consuming homemade crude in Iran has been revealed that among patients with symptoms of paralysis, botulism has been an important differential diagnosis [27]. Tracing diverse types of clostridium botulinum in 4 kinds of food substance via polymerase chain reaction has been revealed through total number 290 specimens the contamination percentages of this bacteria in fish, honey, curd, Dough, were $5 \%, 4 \%, 2.5 \%$, and $1.25 \%$ respectively [28]. It has been reported that quality control over local dairy products is essential to prevent the development of dairy-derived botulinum [29]. Researches by Tavakoli et al. revealed that diverse kinds of fish contamination were the sake of different types of Clostridium botulinum specially A-type. The amount of contamination in processed fishes was nearly 1.5 times in nonprocessed fishes. As the total number of 84 nonprocessed fish specimens (13.09\%) and among a total number of 62 processed fish specimens $(7.57 \%)$ has been demonstrated the presence of botulinum toxin [28]. Studies of Lalitha and Surendran in India [30], Huss in Scandinavia [31], and Nakamura and Yamakawa [32] in Japan have been shown that A-type is the most frequent type of bacteria. Several studies reported that, after this toxin consumed and absorbed in the intestines would be visible muscle paralysis in the respiratory and cardiac system, suffocation, heart failure, and death, the mortality rate is between $30 \%$ and $60 \%$ [33-36]. It has been shown that botulism is caused by a potent neurotoxin that blocks neuromuscular transmission, resulting in suffocation [37]. Gastrointestinal symptoms are common in food botulism (nausea, vomiting, abdominal pain, diarrhea, dry mouth) and may precede neurological syndromes [38]. It has been found that botulism can lead to small bowel obstruction and, if misdiagnosed, can lead to rapid clinical deterioration [39]. Botulinum toxin (BTX) is used in medicine. This toxin is used for beauty, eliminating frowns and facial wrinkles. In addition to its cosmetic effect, neurotoxin has a positive effect on mood and depression treatment [40]. A number of studies found that Suppression of neuropeptide by botulinum toxin improves 
imiquimod-induced psoriasis-like dermatitis via the regulation of neuroimmune system [41]. Botulinum neurotoxin is effective in the treatment of sialorrhea, hyperhidrosis, gastrointestinal dysfunction and urinary dysfunction. Other symptoms of botulinum neurotoxin in Parkinson's disease include pain and movement symptoms such as blephaospasm, cervical dystonia, cervical dystonia, limb dystonia, and tremors [42]. Botulinum toxin is effective in the management of primary and secondary Raynaud's phenomenon [43]. Botulinum toxin type A or anticholinergic drugs are used to treat people with cervical dystonia (involuntary head condition) [44]. Several studies reported that, Botulinum neurotoxin type A has clinical and neurophysiological effects in chronic migraine $[34,45]$. Botulinum toxin (BoNT) is used to treat blepharospasm and other facial spasms, as well as for a variety of neurological and non-neurological disorders. Due to neuromotor disorders, BoNT has been reported to be effective in treating dystonia, gnashing of teeth, tremors, tics, myoclonus, restless legs syndrome, delayed dyskinesia, and a variety of symptoms associated with Parkinson's disease. In addition, the toxin has been developed as a potent muscle relaxant and active marginal drug for pote ntial applications of the central nervous system in the treatment of neurodegenerative disorders [46].

\section{Conclusion}

BoNT is involved in the management and control of many diseases such as depression and Parkinson's, dermatitis such as psoriasis, as well as in the beauty and elimination of facial skin wrinkles.

\section{Ethical considerations}

Ethical issues (including plagiarism, data fabrication, double publication and etc.) have been completely observed by author.

\author{
Competing interests \\ None. \\ Funding statements \\ None. \\ Authors' contribution \\ All authors contributed equally to the \\ manuscript.
}

\section{References}

1. Naseri, M., Rezaei, M., Abedi, O. and Afsharnadari, A.. Measurement of some heavy metals (Iron, Copper, Zinc, Magnesium, Manganese, Mercury, Lead and Cadmium) in edible and non-edible tissues of green back suffocating fish (Liza dussumieri) in Bushehr coast, (2015).

2. Tuzen, M. and Soylak, M. Determination of trace metals in canned fish marketed in Turkey. Food Chemistry. 101(4), 1378-1382(2007).

3. Syd, S.H., Kiai, S.M., Githy, K.,Sidmehdi's spiritual rhizavi, Rekani Nordehr, and Abbas Valie-Marim. Study of studies on contamination of animal-derived foods to pathogenic bacteria in Iran, Part 4: Chicken and Eggs (2013).

4. Moussavi, S.M., Adalat, M., Sadiqi,A., Mohtasham Amiri, Z., Asadi, S., Binesh Barhand, M., Sadiq Kiarod, I., Rezaye, A. and Mahdavi, M.H. Report on botulism cases afflicted by Schaphal Shoor in Rasht city. Journal of Guilan University of Medical Sciences. 16(61), 60-64 (2005).

5. Roberts, R. The pathophysiology and systematic pathology of teleosts. Fish Pathology, (Ed.3), 55132 (2001).

6. Scalfaro, C., Auricchio, B., De Medici, D. and Anniballi, F. Foodborne botulism: an evolving public health challenge. Infectious Diseases. 51(2), 97-101(2019).

7. Nadjafi, M., Knowledge and attitude of Iranian Red Crescent Society volunteers in dealing with chemical attacks. Bulletin of Emergency \& Trauma. 5(2), 122-128(2017).

8. Ghanei, M., Delayed haematological complications of mustard gas. Journal of Applied Toxicology: An International Journal. 24(6), 493-495 (2004).

9. Hellmich, D. Foodborne botulism due to ingestion of home-canned green beans: two case reports. Journal of Medical Case Reports. 12(1), 1-5(2018).

10. Mackle, I., Halcomb,E. and Parr,M. Severe adult botulism. Anaesthesia and Intensive Care. 29(3), 297-300(2001).

11. Murray, P., Rosenthal, K.S, Kobayashi, G.S. and Pfaller, M.A., Medical Microbiology, Arthropods, (2002).

12. Cliver, D.O. and Riemann, H.P. Foodborne Diseases, Gulf Professional Publishing, (2002). 
13. Lindstrom, M. and Korkeala, H. Laboratory diagnostics of botulism. Clinical Microbiology Reviews. 19(2), 298-314(2006).

14. Huss, H.H. and Pedersen, A. C. botulinum Scandinavian waters. Nord. Vet. Med., 31(5), 214222(2011).

15. Huss, H. and Pedersen, A. C. botulinum in scandinavian waters. Biology. 5(2), 214-221 (1979).

16. Ojagh, S.M., Rezaei, M., Razavi, S.H. and Hosseini, S.M. Effect of chitosan coatings enriched with cinnamon oil on the quality of refrigerated rainbow trout. Food Chemistry., 120(1), 193198(2010).

17. Afshani Nagadeh, M. Feature of botulism in Iran. 7th microbiological congress of Iran (bacterial tendency). Semnan, Iran(2004)

18. Rao, A.K. Clinical Guidelines for Diagnosis and Treatment of Botulism, 2021. MMWR Recommendations and Reports. 70(2), 1-30(2021).

19. Semenko, N. Foodborne Botulism in Ukraine from 1955 to 2018. Foodborne Pathogens and Disease. 18(4), 260-266 (2021).

20. Mahdizada, M., Rahimi, I., Peroz, B. and Khajripur, Z. Mururi bar Butulism. Mahanamah Academic P. Zhushi Dnyangah Uloom P.J. P.S. Sadoki Yazd., 10, 16(3), 325-327(2008).

21. Fleck-Derderian, S., Shankar, M., Rao, A.K., Chatham-Stephens, K., Adjei, S. and, Sobel, J.. The epidemiology of foodborne botulism outbreaks: a systematic review. Clin. Infect. Dis., 66(suppl_1), 73-81 (2017).

22. Asl, H.M., Gouya, M.M., Soltan-Dallal, M.M. and Aghili, N. Surveillance for foodborne disease outbreaks in Iran, 2006-2011. Medical journal of the Islamic Republic of Iran. 29, 285, pages 1-6(2011).

23. Khorasan, M.R.M., Rahbar, M., Zahedi Bialvaei, A., Gouya, M.M., Shahcheraghi, F., Eshrati, B._Prevalence, Risk Factors, and Epidemiology of Food-borne Botulism in Iran. Journal of Epidemiology and Global Health, 10(4), 288-292 (2020).
24. Yamasaki, S. Incidence of food borne botulism in Japan during 1977-1998. IASR.;21:241242(2000).

25. Tornese, M. Epidemiology and risk factors associated to foodborne and infant botulism: where and when? Revista chilena de infectologia: organo oficial de la Sociedad Chilena de Infectologia, 25(1),22-27(2008).

26. Cherington, M. Review of Botulism. Semin. Neurol., 24 (2)155-63 (2004).

27. Karbalaei Shabani, A. Type A botulism outbreak in members of a family following consumption of homemade whey: brief report. Tehran University Medical Journal TUMS Publications, 77(11),720723(2020).

28. Sadeghi Sarvestani, Mohammad Vahid, Hossein zadeh and Pourmansari, Fazeli. Traces different types of Clostridium botulinum in 4 types of food using polymeraz chain reaction. J. Fasa Univ. Med. Sci., 2014; 3 (4) :380-386 (2000).

29. Kamaloddini, M.H. and Kheradmand, H.R. A Foodborne botulism Occurrence in Mashhad: Clostridium botulinum in local cheese. Journal of Emergency Practice and Trauma, 7(1), 6668(2021).

30. Lalitha, K. and Surendran,P. Occurrence of Clostridium botulinum in fresh and cured fish in retail trade in Cochin (India). International Journal of Food Microbiology, 72(1-2),169-174(2002).

31. Huss, H.H., Distribution of Clostridium botulinum. Applied and Environmental Microbiology, 39(4),764-769(1980).

32. Yamakawa, K. and Nakamura, S. Prevalence of Clostridium botulinum type $\mathrm{E}$ and coexistence of C. botulinum nonproteolytic type B in the river soil of Japan. Microbiology and Immunology. 36(6), 583-591(1992).

33. Hellmich, D., Wartenberg, K.E., Zierz, S. and Mueller, T.J. Foodborne botulism due to ingestion of home-canned green beans: two case reports. Journal of Medical Case Reports. 12(1), 1-5(2018). 
34. Andreou, A.P. Double-binding botulinum molecule with reduced muscle paralysis: evaluation in in vitro and in vivo models of migraine. Neurotherapeutics, 18(1),556-568(2021).

35. Pfausler, B. Toxin-associated infectious diseases: tetanus, botulism and diphtheria. Current Opinion in Neurology. 34(3), 432-438(2021).

36. Taheri, F. The most important natural antioxidants and native herbs of Iran used in the nervous system as pre-anesthetics. Research Journal of Pharmacy and Technology, 12(3),1453-1456(2019).

37. McNutt, P.M. Neuronal delivery of antibodies has therapeutic effects in animal models of botulism. Science Translational Medicine, 13, 575:eabd7789(2021). doi: 10.1126/scitranslmed. abd7789.

38. Costa, A.M. Foodborne botulism: a case report. Porto Biomedical Journal, 6, 1, e115 (2021).

39. Friziero, A., Da Dalt, G., Baldan, S., Zanchettin, G., Auricchio, P., Gavagna, L., Grego, N., Capelli, G. and Merigliano, S. Foodborne botulism presenting as small bowel obstruction: a case report. BMC Infectious Diseases, 21(1), 1-4(2021).

40. Schulze, J. Botulinum toxin for the management of depression: An updated review of the evidence and meta-analysis. Journal of Psychiatric Research, 135, 332-340 (2021).

41. Amalia, S.N. Suppression of neuropeptide by botulinum toxin improves imiquimod-induced psoriasis-like dermatitis via the regulation of neuroimmune system. Journal of Dermatological Science, 101(1), 58-68(2021).
42. Mitchell, S.D. and Sidiropoulos, C. Therapeutic Applications of Botulinum Neurotoxin for Autonomic Symptoms in Parkinson's Disease: An Updated Review. Toxins, 13(3), 226, pages 1-16 (2021).

43. Ennis, D. Botulinum toxin in the management of primary and secondary Raynaud's phenomenon. Best Practice \& Research Clinical Rheumatology. 107(7), 101684 (2021). DOI: 10.1016/j. berh.2021.101684 PMID: 33965340.

44. Rodrigues, F.B. Botulinum toxin type A versus anticholinergics for cervical dystonia. Cochrane Database of Systematic Reviews. 4, pages 1-30 (2021).https://doi.org/10.1002/14651858. CD004312.pub3

45. Valente, M. Clinical and Neurophysiological Effects of Botulinum Neurotoxin Type A in Chronic Migraine. Toxins, 13(6), 392-393 (2021).

46. Anandan, C. and Jankovic, J. Botulinum Toxin in Movement Disorders: An Update. Toxins, 13(1), 42-44 (2021) 